\title{
Centralization of a regional clinical microbiology service: The Calgary experience
}

\author{
Deirdre L Church MD PhD FRCPC ${ }^{1,2}$, Paula Hall MLT BSc ${ }^{2}$
}

\begin{abstract}
DL Church , P Hall. Centralization of a regional clinical microbiology service: The Calgary experience. Can J Infect
\end{abstract} Dis 1999;10(6):393-402.

\begin{abstract}
Diagnostic laboratory services in Alberta have been dramatically restructured over the past five years. In 1994, Alberta Health embarked on an aggressive laboratory restructuring that cut back approximately $30 \%$ of the overall monies previously paid to the laboratory service sector in Calgary. A unique service delivery model consolidated all institutional and community-based diagnostic testing in a company called Calgary Laboratory Services (CLS) in late 1996. CLS was formed by a public/private partnership between the Calgary Regional Health Care Authority (CRHA) and MDS-Kasper Laboratories. By virtue of its customer service base and scope of testing, CLS provides comprehensive regional laboratory services to the entire populace. Regional microbiology services within CLS have been successfully consolidated over the past three years into a centralized high volume laboratory (HVL). Because the HVL is not located in a hospital, rapid response laboratories (RRLS) are operated at each acute care site. Although the initial principle behind the proposed test menus for the RRLs was that only procedures requiring a clinical turnaround time of more than $2 \mathrm{~h}$ stay on-site, many other principles had to be used to develop and implement an efficient and clinically relevant RRL model for microbiology. From these guiding principles, a detailed assessment of the needs of each institution and extensive networking with user groups, the functions of the microbiology RRLs were established and a detailed implementation plan drawn up. The experience at CLS with regards to restructuring a regional microbiology service is described herein. A post-hoc analysis provides the pros and cons of directing and operating a regionalized microbiology service.
\end{abstract}

\section{Key Words: Microbiology; Regional centralization; Restructuring}

\section{Centralisation d'un service de microbiologie clinique régionale}

RÉSUMÉ : En Alberta, les services de laboratoire diagnostique ont subi une restructuration considérable au cours des cinq dernières années. En 1994, le ministère de la Santé de l'Alberta a entamé une refonte énergique des laboratoires, en vertu de laquelle le budget global habituellement imparti au secteur des services de laboratoire a fondu de près de $30 \%$ à Calgary. Un modèle de prestation intégrée des services a ainsi conduit au regroupement de tous les tests diagnostiques des établissements et de la communauté auprès d'une seule et même société, les Calgary Regional Services (CLS) à la fin de 1996. Les CLS ont été créés par un partenariat entre les secteurs privé et public, soit entre la Calgary Regional Health Care Authority (CRHA) et MDS-Kasper Laboratories. En vertu de sa clientèle et de l'éventail des tests qu'ils effectuent, les CLS offrent des services de laboratoire régionaux complets à la population entière. Les services régionaux de microbiologie au sein des CLS ont été regroupés avec succès au cours des trois dernières années en un laboratoire centralisé de fort volume. Comme ce laboratoire n'est pas installé dans un hôpital, on a établi des laboratoires pour le traitement rapide des demandes dans chaque établissement de soins aigus. Le principe initial qui sous-tend l'éventail des tests proposés dans ces laboratoires rapides est que seuls les tests dont il faut obtenir la réponse en plus de deux heures sont effectués sur place, beaucoup d'autres principes ont été appliqués au développement et à la mise en place d'un modèle efficient et cliniquement pertinent pour la microbiologie. À partir de ces principes directeurs, on a procédé à une évaluation détaillée des besoins de chaque établissement, on a créé un réseau étendu entre les groupes d'usagers, la fonction des laboratoires de traitement rapide des demandes d'analyse microbiologiques et un plan de réalisation détaillé a été conçu. On décrit ici l'expérience des CLS à l'égard de la régionalisation-restructuration du service de microbiologie. Un analyse rétrospective résume les avantages et les inconvénients associés à la direction et à l'exploitation d'un service de microbiologie régionalisé.

\footnotetext{
${ }^{1}$ Departments of Pathology and Laboratory Medicine, and Medicine, University of Calgary, and ${ }^{2}$ Calgary Laboratory Services, Calgary, Alberta Correspondence and reprints: Dr DL Church, Calgary Laboratory Services, 1638 10th Avenue SW, Calgary Alberta T3C OJ5. 
$\mathrm{L}$ aboratory services have undergone a dramatic restructuring within the province of Alberta over the past few years. Changes to the service took place in all regions of the province as a result of major structural changes in the overall delivery of health care in the province. Although change was long overdue, the pace of change in Alberta was largely driven by severe cuts to health care funding. While provincial health care expenditures were decreased by as much as $20 \%$, laboratory service budgets were capped after being immediately reduced by as much as $35 \%$ in the urban regions - the most severe cuts taken by any clinical service. Why were laboratory services the target of such severe funding cuts? In retrospect, clinical laboratories were vulnerable to re-engineering initiatives due to the obvious duplication of services being provided between the public and private sectors, and technological advances that made the throughput more efficient. Laboratory medicine was also not recognized as an important 'clinical' service area because the laboratory did not provide 'hands-on' care to patients. A disproportionate funding reduction, therefore, occurred because diagnostic laboratories were viewed as having no more clinical relevance in the health care system than nonclinical support services such as housekeeping, finance and information technology.

This paper describes the Calgary experience with regards to the restructuring of clinical microbiology services. A post-hoc analysis of the pros and cons of directing and operating a completely integrated and regionalized service is also outlined.

\section{BACKGROUND}

Laboratory services in Alberta were restructured as a result of major changes to the way that public funds were dispersed to provide health care in the province. Alberta Health divided the province into 17 distinct health care regions in 1995. The Calgary Regional Health Authority (CRHA, Region 4) administers and supplies all health care services within a publicly funded system to the city of Calgary and surrounding suburbs. The CRHA also provides many tertiary services to people living in rural health care regions in southern Alberta as well as southeastern British Columbia and southwestern Saskatchewan. These include major adult and paediatric programs for trauma, oncology and bone marrow transplantation, renal dialysis and transplantation, and human immunodeficiency virus (HIV)/AIDS care. Most of the 1950 physicians in the region provide primary care, but there are also specialists and subspecialists in the major areas of medical and surgical practice.

The CRHA dramatically reduced the overall number of acute care hospital beds in Calgary within the past five years because, in 1994, the system was overbuilt compared with the populace it served. Before restructuring, Calgary had seven major acute care hospitals including the Foothills Medical Centre (FMC), the Alberta Children's Hospital (ACH), the Grace Women's Hospital, the Calgary District Hospital Group (Holy Cross Hospital and Rockyview General Hospital [RGH]), and the Bow Valley Centre (Calgary General Hospital and the Peter Lougheed Centre [PLC]). The Grace Women's Hospital was relocated to the North Tower at FMC. The Holy Cross Hospital was closed in 1996 and put up for sale. A demolition crew im- ploded the Calgary General Hospital in 1998. Hospital closures of this magnitude required the major relocation of several patient care programs to the remaining adult hospitals in the region. For example, the trauma, neurosciences/neurosurgical and cardiovascular programs were amalgamated from all other acute care sites and transferred to the FMC. To accommodate such a major downsizing of acute care hospital space, all services that could be delivered in the community were moved off-site (ie, administration, financial services, information technology services, etc). Due to the dramatic, unpredicted growth in the population in Calgary over the past three years (approximately 110,000 people), the acute care system currently operates at over $90 \%$ capacity, and it is likely that a new hospital will need to be built within the next few years.

Before the restructuring of laboratory services, Alberta Health had different ways of paying the public and private sector providers. Each hospital had its own full service laboratory that was funded through the global operating budget of the facility. Typically, laboratory support services such as finance, human resources and housekeeping were provided as part of the hospital budget and were not included in the laboratory budget. This meant that the laboratory had a capped operating budget and that it competed with all other hospital services for available monies. Capital funds for hospital laboratory equipment came from the general hospital capital budget, and in part, from a provincial fund established to pay for laboratory equipment. Under this payment system, there was disparity based strictly on the clinical service workload (ie, volume of tests) done between the hospital laboratory operating budgets among various facilities. In general, the laboratories located in the academic teaching hospitals were funded at a higher cost per test than the community hospitals. This was arguably due to the fact that more of the highly complex and specialized tests were performed at the academic centres as well as the unique teaching and research responsibilities of these sites. In the community, four private laboratories provided services to physicians' offices and clinics that collected specimens via 110 patient service centres (PSCs). The 'private' or independent laboratories were also being paid by public monies through direct billing on a per test or fee-for-service basis. The large amounts of revenue generated by the private laboratories were justified in part because they were responsible for all operating as well as capital expenses.

All public and private laboratories in the region operated independently of one another so that there was little standardization of policies and procedures across the region. The lack of compliance with recognized laboratory standards in many instances produced a widely variable quality of service, not only among acute care hospital laboratories but also among the private community-based laboratories. There was competition for customers, and individual laboratories continually sought to expand not only their volume of work but also the scope of tests performed. This resulted in a duplication of services and a gross excess of overall capacity in all laboratory disciplines.

In 1995, only two of the hospital laboratories, the FMC and 
$\mathrm{ACH}$, had affiliation agreements with the University of Calgary, where most teaching and research activities in laboratory medicine took place. The Department of Pathology had an accredited residency training program in anatomic pathology, and the Department of Microbiology and Infectious Diseases had a residency program for medical microbiology. In addition, residents in internal medicine, general pathology and other specialties rotated through the microbiology laboratory as did fellows in the infectious diseases training program.

Alberta Health embarked on an aggressive laboratory restructuring process five years ago, and by last year had cut back approximately $35 \%$ of the overall monies previously paid to the laboratory service sector in Calgary. Before these cutbacks, the total annual operating expenditures for clinical laboratory services, excluding capital investments was about $\$ 97$ million. In contrast, the operating budget in fiscal 1998 was $\$ 67$ million, which included all capital and operational expenses. Before 1995, quarterly or biannual cuts of as much as $5 \%$ to $10 \%$ were made to hospital laboratory budgets starting in 1993. Within the private laboratory sector, major consolidation was driven in 1995 by government withdrawal of the fee-for-service or E-code billing schedule. By the end of 1995, a consolidated community-based laboratory called MDS-Kasper Medical Laboratories had emerged as the sole service provider in this sector. A single centralized laboratory site provided all services to the community, and most of the patient service centres had been closed. Only 25 PSCs remained in the region, and these were redesigned and relocated to be strategically located, close to major transportation routes and areas where medical practice was concentrated.

Systematic severe budget cuts necessitated the development of a new laboratory service delivery model for the region. In 1995, an external firm, Chi-Consultants, Ann Arbour, Michigan, was hired by the CRHA to consult with regional laboratory service providers to propose a business solution that could meet the new funding targets. Early in 1996, a final business plan was developed and submitted to Alberta Health; this plan outlined the desired final state of the restructure of laboratory service in Calgary under a proposed consolidated model for the region. The central principle that formed the basis of the new regional service delivery model was that all tests with a turnaround time of more than $2 \mathrm{~h}$ would be moved out of hospitals into a centralized high volume laboratory. Only STAT or urgent tests that required immediate results would be left in hospital rapid response laboratories (RRLS). This meant that virtually all clinical microbiology analytic services would no longer be performed at on-site laboratories, but that specimens would be transported for testing to a centralized high volume laboratory located in the community.

\section{FORMATION OF CALGARY LABORATORY SERVICES}

In Calgary, several restructuring models were considered for the delivery of laboratory services before adopting a solution that incorporated all major sectors, with the exception of the Canadian Blood Services and the Southern Alberta Provincial Laboratory of Public Health. The Calgary Laboratory Services (CLS) service delivery model has been recognized as being unique in North America. CLS was formed in late 1996 as a public/private partnership between MDS-Kasper Medical Laboratories, which was the remaining private provider, and the laboratory subsidiary of the CRHA, which comprised all laboratories within acute care hospitals and many long term care facilities. By virtue of its customer service base and scope of testing, CLS uniquely provides comprehensive laboratory services to an entire region's population, be it inpatients, the institutionalized elderly or ambulatory patients in the community. CLS also provides laboratory services to several rural regions in southern Alberta. Although the University of Calgary is not a partner in the company, an affiliation agreement has been signed with CLS. This allowed many key medical staff members to retain geographic full-time academic appointments with the University of Calgary and provided an essential academic focus for CLS.

\section{RESTRUCTURING OF MICROBIOLOGY SERVICES WITHIN CLS}

Microbiology services were put under extraordinary pressure to restructure in as short a timeframe as possible once CLS was created (November 1, 1996). Not only had dramatic funding reductions required that efficiencies were rapidly realized, but also no other option for hospital microbiology services remained other than to centralize analytic services off-site immediately.

Space: The CRHA began to demolish existing on-site laboratories at each of the remaining adult hospitals (ie, RGH, PLC and FMC) in the year leading up to the formation of CLS, leaving much smaller on-site laboratory spaces called RRLs. As each RRL space was completed, it was expected that most laboratory services (ie, microbiology) that did not require a STAT turnaround time would be moved immediately. The lack of coordination between the RRL construction schedules and the formation of CLS occurred because of the pressure created by planned hospital closures in the region, and because there was a delay in the approval of the Calgary restructuring model for laboratory services.

RRL construction within each adult hospital was completed at staggered times. The RRL in the RGH was completed in December, 1996, followed by the RRL at the PLC in April, 1997. The RRL at the FMC was not completed until September 1997 because construction started much later at this site due to the complex nature of some of the tests performed there, such as flow cytometry, special chemistry and centralized blood bank. Although a separate RRL was not constructed at the $\mathrm{ACH}$, the laboratory there was consolidated within its existing space.

Although the final layout of the RRLs is somewhat different, a central accessioning area occupies the front of each RRL to accommodate sending out several hundred specimens a day to the high volume laboratory. The remainder of the RRL space is mainly designed to handle STAT testing in chemistry and hematology. Space is also allocated to tissue processing for surgical pathology STAT frozen sections with adjacent office space for the pathologist(s). Approximately $23.7 \mathrm{~m}^{2}$ of RRL space was left in each hospital as a microbiology service area. 
TABLE 1

Principles guiding the establishment of rapid response laboratories (RRL) test menus and functions at Calgary Laboratory Services

\begin{tabular}{|c|c|}
\hline Guiding principles & Operation/clinical service/implications \\
\hline $\begin{array}{l}\text { 1. Microbiology analytical work would be centralized to the high } \\
\text { volume laboratory }(\mathrm{HVL}) \text { unless a preliminary or final result was } \\
\text { needed in } 2 \text { h or less. }\end{array}$ & $\begin{array}{l}\text { - All STAT procedures had to be done at the RRL as required by the } \\
\text { clinical services at each hospital. }\end{array}$ \\
\hline 2. All specimen accessioning would be done at the HVL. & $\begin{array}{l}\text { - Specimen handling of critical specimens would be delayed, as would } \\
\text { the overall test turnaround time if all accessioning was done at the } \\
\text { RRL. }\end{array}$ \\
\hline $\begin{array}{l}\text { 3. All specimen quality assurance activities, such as rejection of } \\
\text { improperly collected specimens, would be done at the rapid } \\
\text { response laboratory (RRL). }\end{array}$ & $\begin{array}{l}\text { - Immediate rejection of poor quality specimens and request for } \\
\text { recollection would minimize the test turnaround time. The HVL would } \\
\text { also not have to deal with inappropriate and/or improperly collected } \\
\text { hospital specimens. }\end{array}$ \\
\hline $\begin{array}{l}\text { 4. Total transport time from the site of collection to the HVL should be } \\
\text { no longer than } 2 \mathrm{~h} \text { for specimens not in a transport medium. }\end{array}$ & $\begin{array}{l}\text { - A complex transportation system had to be established to get } \\
\text { specimens from the hospital RRL accessioning areas to the HVL. }\end{array}$ \\
\hline $\begin{array}{l}\text { 5. Laboratory assistants needed to be able to handle all types of } \\
\text { specimens proficiently. }\end{array}$ & $\begin{array}{l}\text { - Within the RRL and HVL central accessioning areas had to be } \\
\text { cross-trained to handle microbiology specimens. }\end{array}$ \\
\hline $\begin{array}{l}\text { 6. Microbiology RRLs and the HVL had to be staffed by highly trained } \\
\text { and experienced technologists. }\end{array}$ & $\begin{array}{l}\text { - Technologists would not be cross-trained from other laboratory areas } \\
\text { to perform microbiology procedures. }\end{array}$ \\
\hline $\begin{array}{l}\text { 7. Specialized tests for fastidious organisms would be centralized to the } \\
\text { RRL as dictated by clinical need. }\end{array}$ & $\begin{array}{l}\text { - Pertussis cultures would be centralized to the Alberta Children's } \\
\text { Hospital. }\end{array}$ \\
\hline 8. A regional laboratory information system (LIS) would be required. & $\begin{array}{l}\text { - The selected LIS would have to have multisite capability so that } \\
\text { specimens could be tracked and reported from all service sites. The } \\
\text { laboratory information service must have a comprehensive } \\
\text { microbiology module. }\end{array}$ \\
\hline
\end{tabular}

This space contains minimal bench space to house a microscope and a computer terminal, a biohazard hood in which specimens may be separated and handled, and a sink for performing Gram stains. In the end, the $1894 \mathrm{~m}^{2}$ of space that had existed for on-site hospital microbiology services were consolidated to approximately $94.7 \mathrm{~m}^{2}$ of space within the RRLs. All hospital microbiology services were centralized to one of the existing community laboratory sites in downtown Calgary. The high volume laboratory currently operates in approximately $568.2 \mathrm{~m}^{2}$ of space. This has created an extremely compact environment that does a very large volume of testing and accommodates more staff in less than one-third of the space previously occupied by clinical microbiology services in the region. However, the current space is clearly inadequate for the size of the current CLS microbiology operation and does not allow room for growth and expansion. A new facility, which will contain significantly increased and properly designed space for regional microbiology services, will be built for the high volume laboratory within the next two years.

Centralization of microbiology services: Centralization of microbiology services had to be carefully planned and organized to ensure that patient care was not compromised during the process. The underlying principle was that all infrastructure, resource and clinical issues had to be addressed and resolved before the movement of any patient tests from the hospitals.

Establishing the RRL test menu and functions: In anticipation of regional restructuring, an implementation group was formed in 1995 that comprised medical microbiologists, managers, supervisors and senior technologists who represented each hospital laboratory as well as the private community- based laboratories. This group of experts laid out the key operations of a microbiology RRL. Table 1 outlines the principles used along with the major operational and clinical service issues addressed to establish the hospital RRL test menu and functions. A comprehensive assessment of the clinical services located at each hospital was completed to determine which microbiology tests and functions needed to remain at each hospital RRL. For example, as the major tertiary academic paediatric care centre, the ACH needed rapid diagnosis for rotavirus and respiratory syncytial virus (RSV) infections. The RRLs of the two community general hospitals, RGH and PLC, performed most of the orthopedic joint replacement surgeries, so that the RRLs at those hospitals had to be able to provide 'superSTAT' (ie, within 30 mins) Gram stain results on harvested tissue biopsies during prosthetic joint reimplantation and septic trauma cases. Special procedures were implemented for handling synovial fluids at the RRL at the RGH where the rheumatology unit for the city was located. Specialized protocols for efficiently handling all types of fluids and tissues that needed urgent analysis by several laboratory disciplines (ie, chemistry/hematology) aside from microbiology were also put in place. For example, special procedures were developed for handling cerebrospinal fluid and bronchoalveolar lavage (BAL) fluids. Consultation was also sought with key physician user groups including members of the Division of Infectious Diseases, University of Calgary so that the RRL test menus and services proposed were reviewed by these groups before being finalized.

Table 2 outlines the microbiology RRL test menu and functions that were proposed by the implementation group. Aside from a few specialized tests that were unique to paediatric 
testing (ie, ACH), all of the other RRLs had similar service needs. Initially, urgent stains for Pneumocystis carinii pneumonia detection in bronchoalveolar fluids, and thin and thick smears for malaria were done exclusively at the FMC's RRL. However, these regional services have since been consolidated at the high volume laboratory where trained technologists can be most efficiently scheduled to cover the service demands after daytime hours. In addition, as implementation of each RRL proceeded, it became more efficient to move certain specimen quality assurance procedures (ie, sputa Q-scores) to the high volume laboratory.

The hospital microbiology RRLs are staffed daily between 08:00 to $16: 15 \mathrm{~h}$ by a highly experienced technologist who is familiar with the unique operations and clinical environment of that site. Although technologists are rotated between the RRL and the high volume laboratory, staff have not been rotated between the various RRL sites. This policy has ensured that the RRL technologist has been able to act as an effective clinical liaison with clinicians and other health care workers within each hospital.

Front-end issues: Inadequate front-end infrastructure and resources can "kill you" in centralizing and operating a microbiology laboratory the size of CLS. The pre-analytic processes are more complex than any other laboratory area not only because of the variety of specimen sources and types handled, but also because specimens are collected by a multitude of personnel including patients. Standardized policies and processes had to be put in place for the collection, transportation, accessioning and planting of specimens within CLS. Because of the unique operations that existed within each hospital as well as the community, a full-time technologist was dedicated to this 'front-end' project for over a year. This person also became responsible for training personnel in the accessioning and microbiology areas about front-end practices. Collection devices and containers were standardized throughout CLS and allowed physicians to collect microbiology specimens from both ambulatory as well as hospitalized patients using the same system. A standardized clinical collection method for every type of sample or test replaced all other existing procedures and formed the basis of the CLS guide to services. A consolidated 'green' microbiology requisition was designed and implemented, and replaced the 17 different requisitions that had been in use in the hospitals and community laboratories. The FMC is currently the only hospital that has an information system in place that allows the electronic order entry of laboratory tests.

Sterile separation of microbiology specimens that require an aliquot to be sent to another laboratory area (ie, cytopathology) is done at the RRL, but all specimen planting is done at the HVL. Centralization of accessioning and planting allows media to be stocked and replenished at the high volume laboratory. Due to the high staff turnover in accessioning in the RRLs that occurred at the start of restructuring, it was not possible to maintain enough expertise in microbiology. It was, therefore, more efficient to staff a centralized accessioning and planting area with highly trained laboratory assistants and technologists who could competently handle any type of critical microbiology sample. Another major factor in deciding
TABLE 2

Microbiology rapid response laboratory functions and test menus* at Calgary Laboratory Services

- Initial specimen accession for tracking purposes

- Specimen quality assessment

- Specimen rejection of inappropriately collected or poor quality specimens

- Specimen separation and aliquotting

- Packaging and shipping of tests to a reference laboratory

- STAT or urgent Gram stains (ie, sterile fluids and tissues)

- Urinalysis

- Cell counts and chemistry analysis of sterile fluids (ie, cerebrospinal fluids and tissues)

- Specimen tracking of overdue reports

- Bordetella species cultures (Alberta Children's Hospital)

- Rapid virology (rotavirus/respiratory syncytial virus) (Alberta Children's Hospital only)

- Clinical liaison to users at the hospital

- Communication to the high volume laboratory

- Entry of reports into the hospital LIS ${ }^{+}$

- Hard copy report sorting for distribution

*All functions and tests are performed at each RRL unless otherwise indicated; ${ }^{+}$Before the installation of regional laboratory information system (LIS)

not to plant specimens in the RRLs was the widely variable climatic conditions in the region at different times of the year. The average daily temperature in Calgary in the summer months is $22^{\circ} \mathrm{C}$, but in the winter, the daily temperature can dip below $0^{\circ} \mathrm{C}$ with very little notice. Although insulated specimen carrier boxes were available, transportation of a large volume of inoculated agar plates that had to be maintained under appropriate environmental conditions would have added another layer of complexity to the system.

CLS took the approach that it would be better to develop a very efficient external transportation system that could deliver critical specimens from any hospital site to the RRL within 1 to $2 \mathrm{~h}$ of collection as required. Expertise gained from the private laboratory sector designed a tightly coordinated external transportation system that could move a large volume of critical microbiology specimens around the region of Calgary. CLS employs a fleet of 20 courier vehicles that follow designated routes among the four hospitals, PSCs and physician offices so that all available specimens that have been accessioned onto the computer transport tracking list are immediately sent on the next courier run. Two CLS drivers are dedicated to STAT pick-up of specimens from the hospital RRLs. In addition, critical samples that arrive at the RRL in between courier runs can be transported by priority taxi service. CLS routinely tracks the time taken during the pre-analytic phase for various processes and audits any critical specimens that arrive late. Most often delays in transportation occur between the time of collection and receipt of critical samples in the RRLs. Because hospital porters and other CRHA personnel are involved in conveying microbiology specimens from the wards to the RRLs, CLS currently has very little control over improving transport delays within the hospitals. 
TABLE 3

Specimen priority for movement of microbiology work

\section{Noncritical specimens*}

- Stool for culture

- Stool for ova and parasites

- Environmental specimens

- Mycology (superficial hair, skin, nails)

- Urogenital specimens (urethra, vaginal, cervix)

- Upper respiratory specimens (eye, ear, nose, throat)

- Outpatient urines for culture and susceptibilty (excluding emergency room specimens) (a)

Critical specimens ${ }^{+}$

- Blood cultures

- Sterile fluids

- Specimens from surgical patients

- Specimens from critically ill patients (to be defined at each hospital)

- Tissues

- Deep wounds/abscesses/aspirates

- Lower respiratory track specimens (sputum, bronchial washes, bronchoalveolar lavage)

- Catheter tips/sites

- Nonoutpatient/emergency roomurines

- Mycology (nonsuperficial)

- Specimens from Eemergency room patients

- Specimens from patients in any ICU

- Specimens from patients admitted to oncology/burn unit

- Superficial wounds (excludes line sites and all ICU, emergency room, oncology specimens)

- Stool for Clostridium difficile (dependent on the adoption of a common procedure)

* Specimens already performed in community laboratory that could be recollected; ${ }^{+}$Invaluable specimens that could not be easily recollected and specimens from critically ill (ie, intensive care unit [ICUI) patients

Movement of specimens: Microbiology services were moved offsite to the high volume laboratory from each hospital in a staggered fashion. Analytic microbiology work was moved according to the level of medical priority given to specific types of specimens. Table 3 outlines the 'noncritical, can be recollected' versus 'critical, invaluable specimen' categories that guided specimen transfer. Because the hospital work was merged into an existing community laboratory operation, 'noncritical, can be recollected' tests that were already being routinely performed were moved first. This approach also ensured that all of the required infrastructure, policies and procedures were operational before attempting to move tests affecting patients who were critically ill (ie, those in intensive care units [ICUs]). 'Critical, invaluable specimens', such as those from ICU patients, were only moved after all operational problems had been satisfactorily resolved from a medical perspective. Because critically ill patients often require coordination of the analytic work, all critical specimen types (ie, blood and sterile fluid cultures, etc) were moved as a block so that communication about key test information on the sickest patients was not compromised.

Hospital work from each site was moved and consolidated into the high volume laboratory at staggered times. The merger of each hospital laboratory was completed as much as possible before work was moved from the next site. For the most part, construction schedules for the RRLs dictated the timeline for movement of microbiology services. The RGH laboratory was totally consolidated into the high volume laboratory by January 1997 followed by Calgary General Hospital (April 1997), ACH (September 1997) and FMC (January 1998). The key quality indicators and tolerance limits monitored throughout the merger process for each hospital site included: turnaround times for different tests; critical incidents that affected patient care and/or outcome whether due to error within or external to the laboratory; and all specimen problems relating to the collection, transport or accessioning of any microbiology specimen.

High volume laboratory operations: Before restructuring, hospital microbiology laboratories for the most part operated during daytime hours with limited service provided by callback for critical procedures after 16:00. Only the FMC laboratory was staffed during the evening shift until 23:00 on weekdays. The CLS centralized high volume laboratory operates $24 \mathrm{~h}$ a day, every day of the year, which allows for improved test turnaround times for many types of specimens. Highly trained technologists are also readily available to perform STAT procedures for specialized regional services such as $P$ carinii stains on lower respiratory specimens, and thin and thick smears for malaria.

Round-the-clock operation not only allows the most efficient use of space and staff, but also is necessary to meet the clinical needs of critically ill patients. Because there are only three adult hospitals in the region operating at maximum capacity, the current situation demands that a full-time service be available. For example, some operating rooms function $24 \mathrm{~h}$ a day, seven days of the week. Due to the serious level of acuity of programs and patients that is concentrated at the FMC, invasive procedures are also frequently performed during the evening or at night. If laboratory services had not been centralized, hospital microbiology laboratories would now need to be staffed at some level $24 \mathrm{~h}$ a day to meet the clinical demands of the newly restructured health care system in Calgary.

Standardization of service delivery: To consolidate hospital services into a community laboratory operation, policies and procedures had to be standardized within CLS. This was a large task because none of the hospital or private laboratories performed testing in exactly the same way. Standardization committees were struck across the region for every area of microbiology testing. Each committee comprised technologist staff from the community laboratory and each hospital site. The regional manager for CLS microbiology services and/or one of the two supervisors also participated. These committees were charged with reviewing each laboratory's previous policies and procedures as well as the literature so that new 'best practices' documents could be drafted. Methods were changed to comply with available laboratory standards (ie, National Committee NCCLS) wherever possible. The Clinical Leader, Microbiology not only reviewed all of the draft technical documents, but also addressed all of the outstanding medical concerns. Deficient service areas were targeted for development or improvement wherever 'holes' in service had previ- 
ously existed. Although all hospital sites were performing testing on BAL, little standardization of clinical or laboratory practice as to how these critical samples should be tested existed. A physician's user group that included key stakeholders from each hospital (ie, respirologists and intensivists) was struck by the Clinical Leader, Microbiology. This group of experts put in place a comprehensive regional policy for all aspects of BAL culture. Once all of the medical issues had been satisfactorily addressed, each document was signed off by the Clinical Leader, Microbiology and senior technical staff, and functioned as the first draft of the CLS Microbiology Laboratory bench manual.

As a simultaneous process, an inventory was carried out of all microbiology equipment in the region. As part of the standardization and consolidation process, key pieces of equipment had to be replaced. Although all of the hospitals used VITEK (bioMérieux Canada, Inc, St-Laurent, Québec) as the major laboratory analyzer, the community laboratory used the Replicator system (Oxoid, Nepean, Ontario). Because the Replicator system database could not proficiently identify many nosocomial pathogens, and antibiotic susceptibility tests took 24 to $48 \mathrm{~h}$ to produce results, a decision was made to use VITEK as the major analyzer for all CLS testing, and new instruments were purchased. This approach allowed identification and susceptibility results from ambulatory and hospital patients to be provided in a more timely fashion, ie, same day. A custom 45-well antibiotic susceptibility card was designed that would accommodate the reporting of predominantly oral antibiotic choices for ambulatory patients and parenteral antibiotic choices for inpatients.

Blood cultures were consolidated at the high volume laboratory into a bank of BacT-Alert (Organon-Teknika, Durham, North Carolina) instruments. An analysis was done of the pros and cons of leaving blood culture instruments distributed at each hospital site versus centralizing all testing to the high volume laboratory. Centralization of the blood culture instruments offered many operational, technical and clinical advantages. Due to the extended hours of operation of the high volume laboratory versus the RRL sites, positive blood cultures could be analyzed as soon as they were flagged by the instrument. If the blood culture instruments had been left at the hospital RRLS, there would have been a significant delay in analysis because the bottle(s) would need to be transported to the high volume laboratory. Because patients are frequently transferred between hospitals in the region, all physician phone inquiries could be referred to the central laboratory to maintain clinical continuity. Centralization also made staffing of the blood culture service more efficient and required a lower number of instruments and computers. Blood culture statistics for the entire region could also be more easily maintained and retrieved from a central database.

The major cons identified were the potential delay in initial incubation of bottles that could occur if external transport from the RRLs to the high volume laboratory was compromised. However, this was not a big concern because the blood culture instruments could handle a delayed entry of up to $24 \mathrm{~h}$ and the inoculated bottles would be incubated at $35^{\circ} \mathrm{C}$ at the
RRLs before transport. A large amount of space would be needed at the high volume laboratory, with dedicated electrical input, to house the estimated eight to 10 individual cabinets that would be needed to accommodate the region's current and projected five-year blood culture bottle volume. However, in 1997, CLS implemented a four-day incubation protocol for blood cultures based on previously published work from the FMC site (1). This protocol has allowed the 5000 blood cultures a month to be accommodated within six BacTAlert cabinets.

All supplies including media, reagents, supplies and disposables were reviewed so that purchasing could be consolidated, in most cases, with a single supplier. Enough inventory is only kept on-site at the high volume laboratory to meet the operational needs of two weeks' work. The supervisor and a full-time laboratory assistant are responsible for regular inventory of stores and coordinating orders through purchasing to ensure that the laboratory never runs short of anything.

Human resources issues: The magnitude of change faced by all personnel and the timeframe in which change occurred was truly remarkable. One of the most agonizing aspects of restructuring has been witnessing how different people and personalities adapt to the stresses and challenge of massive change. In retrospect, we equate the transformation at CLS with a fast moving train. Some people got on board from inception, rose to the challenge and have thrived in this fast-paced high volume environment. For a variety of reasons, other people needed to get off the train along the way. One of the biggest challenges faced by management was establishing and fostering new teams. First and foremost, the diverse expertise and skills that laboratory assistants and technologists from the various sectors brought to the company were respected. Although hospital technologists were expert at performing complex tests, some had difficulty adapting to the amount and pace of the work in the high volume laboratory. In contrast, community-based technologists for the most part had limited or no experience performing certain types of complex hospital tests, but they were expert at efficiently handling routine high volume work. It also took time for staff from each hospital site to become a coordinated unit. Each successive hospital merger created the need to redesign teams to integrate expertise with a unique perspective.

Several processes helped the staff to integrate more quickly. Hospital staff from each site were rotated through the high volume laboratory so that they could be trained to use the existing laboratory information system. Also, once a policy, procedure or method was standardized, it was immediately implemented not only at the high volume laboratory, but also at each of the RRL sites if the work was still being performed at that RRL. All staff was brought under a single union contract within CLS. Before completion of these negotiations, all hospital and community technologists had been covered under the Health Sciences Association of Alberta (HSAA). Laboratory assistants belonged to the Canadian Union of Public Employees and Amalgamated Union of Public Employees in the hospitals and HSAA in the community. All staff became part of HSAA as a result of negotiations with CLS, with sub- 
stantial improvements in salary and benefits in many cases. No unionized staff were laid off because CLS made an open voluntary severance package available to staff who applied for the first year after the company was formed. Through this mechanism, up to one-third of the existing regional laboratory assistant and technologist staff pool in microbiology were 'severed'. Nonunionized staff did not fare as well. CLS initially hired only one manager and two supervisors through a competitive process. Following consolidation, management has been further reduced to one manager and a single supervisor for microbiology.

One of the key requirements for maintaining quality microbiology services in the region was the maintenance of competent staff. The union agreed that personnel had to have a minimum of one year of dedicated microbiology laboratory experience, and that experience had to have occurred within the past five years. This meant that staff working in other laboratory areas with more seniority but no microbiology experience were prevented from displacing highly qualified personnel who had many years of dedicated microbiology expertise. Following consolidation, a microbiology experience survey was conducted by management. CLS has over 200 collective years of experience within its laboratory assistance pool, many staff have Level II training in microbiology, and there is more than 1000 years of collective expertise among the CLS technologist pool, with many staff having Advanced Registered Technologist and/or Bachelor of Science degrees. The retention of an extremely qualified and dedicated staff was key in maintaining a quality microbiology service throughout this period of tremendous change.

Communication: Centralization of microbiology services meant that hospital physicians could no longer visit the microbiology laboratory on rounds. Severing of face to face interaction in the hospitals among clinicians and the laboratory staff was one of the most difficult transitions that everyone had to make. However, before restructuring, several hospitals in Calgary operated with off-site microbiology laboratories. For example, the Bow Valley Centre (Calgary General Hospital and the PLC) had all microbiology services provided by the laboratory at Calgary General Hospital. In addition, the microbiology laboratory had been moved out of the main hospital building into the basement of an adjacent building at the FMC in 1992, thereby limiting easy access to busy physicians.

Communication by other means had to be enhanced in the new system. CLS operates a Laboratory Information Centre (LIC) at the high volume laboratory that is staffed during extended hours. LIC operators are expert at communicating laboratory results that have been entered into the laboratory information system. In addition, LIC triages call for referral to specific bench areas in the microbiology laboratory and to the microbiologists. Telephone access is also available directly into microbiology and to specific bench areas (ie, blood cultures) through several central numbers. Physicians can immediately communicate with a technologist about the latest test analysis information as required. In the evening, technical staff carry a mobile telephone so that calls are not missed. The medical microbiologist on call reviews all positive culture in- formation daily and communicates with physicians wherever necessary to obtain additional clinical information, to relay critical specimen results and to rectify any identified problems or concerns about an individual's specimen and/or test(s). The Clinical Leader is also available by pager to address immediate service concerns.

In the future, new ways will be found to communicate laboratory results to physicians. Telemedicine offers many exciting uses of technology, allowing medical services to be delivered to the point of need (2). Rapid advances in computer and telecommunication technology, particularly the Internet, are making it possible to provide laboratory consultation in a real time fashion through interactive video, or asynchronously through the transmission of text, graphics, auditory verbal information, still images and short video clips (2-5). Electronically generated microbiology reports could contain not only text but also pictures of the microscopic examination results (ie, Gram stain), video clips of the growth of the organism on primary culture media and the visual results of biochemical tests, or even full-motion video of the organisms in motion (ie, helminths). This type of reporting would not only convey more information to the clinician, but also greatly enhance the educational ability of the microbiology service. If the trend to the centralization of laboratory services within regions or between regions continues, it is likely that telepathology services will proliferate and provide a new way of providing consultation as well as expanding the access and availability to complex services across regions (5).

Laboratory information system issues: Centralization of laboratory services in the long term would not be sustainable without the acquisition and implementation of a regional (laboratory information system) LIS and the support of an expert information systems staff. A comprehensive LIS selection process was undertaken in the year before the formation of CLS to complete the necessary request for proposal process, review available systems and finalize the selection of a vendor. The result of this process was that the PathNet Classic 306 system (Cerner Corporation, Kansas City, Missouri) was purchased by CLS for development and installation as the regional LIS solution.

Before activation of PathNet, CLS used multiple legacy systems that required support. The microbiology high volume laboratory used MEWS (MDS, Autolab, Toronto, Ontario) interfaced to an AS400 server system (IBM, Toronto, Ontario). MEWS allowed the microbiology laboratory to be relatively paperless because all work was entered into the system by the technologist. Because microbiology work had to be moved before implementing PathNet, a different solution had to be worked out for each hospital to maintain seamless results reporting from the legacy systems. For the two hospitals that had LIS systems (FMC and PLC), computer terminals from each system were established at the high volume laboratory through modem connections. Each of these hospitals also had hospital information systems (HIS) that were interfaced with their respective LIS systems, but only the HIS at FMC allowed electronic order entry for laboratory tests. To make the operation as seamless as possible to clinicians, PLC/FMC technolo- 
gists have re-entered microbiology results from MEWS back into their respective LIS systems. This allowed microbiology laboratory results to be electronically transmitted back to these sites so that reporting delays were minimized. At the $\mathrm{ACH}$, the VITEK AMS computer system was used to store results, and to generate patient reports and statistics. Technologists have also maintained the database at $\mathrm{ACH}$ by re-entering results. Because no LIS was in place at the Rockyview site, CLS organized the print and report distribution times off the central MEWS/AS400 system to print at the RRL in advance of regular laboratory report pick-ups by hospital porters.

Two microbiology technologists were dedicated to the PathNet Microbiology Implementation project full time for the past two years. They have been responsible for working with the Microbiology Acceptance Team and an expert user group in microbiology to customize the development of the microbiology module to meet the complex demands of the CLS operation. Although trained information services personnel could have been assigned to this task, it was much more efficient to assign microbiology technologists who understood fully how the laboratory operated than to try and teach an information services person the laboratory business. This approach has been extremely effective in successfully completing the development of the LIS and implementing it with minimal problems. Implementation of PathNet will allow CLS to turn off all other existing legacy systems and to stop re-entering results into these hospital systems. In addition, all major instruments have bidirectional interface to the PathNet system that allows the automatic upload of batch-negative blood culture results to the LIS as well as the seamless transfer of antibiotic susceptibility results from the VITEK analyzer. This has made the laboratory workflow much more efficient. PathNet also has sophisticated data retrieval and analysis capabilities that are being developed so that aggregate data can be readily compiled (ie, antibiograms). In addition, the system can produce comprehensive reports about quality indicators (ie, test turnaround times, front-end processed turnaround time, quality control, etc). To enhance communication to major surveillance programs in the region, the PathNet Infection Control module was developed and implemented in a collaborative project with the users (ie, Acute Care Regional Infection Control Program, Population Health, Community Health Resources). Candidate reports routinely extract and allow the daily electronic transmission of a large amount of surveillance data from the CLS microbiology high volume laboratory. For example, candidate reports provide an aggregate report of patients who are carriers or infected with an antibiotic-resistant organism to all hospital infection control offices. Population Health also receives an aggregate report of all patients with potential or confirmed notifiable communicable diseases. Aggregation of regional laboratory data at CLS has allowed nosocomial (ie, vancomycin-resistant enterococci) and community-based outbreaks (ie, enteric) to be detected rapidly.

Academic activity: The CLS model has created an environment for enhanced academic activity. Centralization of service has created a large microbiology database that is representative of the entire populace in the region as well as several ex-
TABLE 4

Pros and cons of centralized microbiology services

\begin{tabular}{|c|c|}
\hline Pros & Cons \\
\hline $\begin{array}{l}\text { - Standardized processes and } \\
\text { procedures }\end{array}$ & $\begin{array}{l}\text { - Need tightly coordinated } \\
\text { implementation effort sustained } \\
\text { over several years }\end{array}$ \\
\hline - Efficient, integrated service & $\begin{array}{l}\text { Face to face interaction with } \\
\text { medical staff limited in off-site } \\
\text { laboratory }\end{array}$ \\
\hline $\begin{array}{l}\text { - Improved quality of continuous } \\
\text { service delivery to hospitals } \\
\text { ( } 24 \text { h each day, seven days a } \\
\text { week) }\end{array}$ & $\begin{array}{l}\text { - Need to implement enhanced } \\
\text { electronic reporting and medical } \\
\text { consultation capabilities (ie, } \\
\text { telemedicine/telepathology }\end{array}$ \\
\hline $\begin{array}{l}\text { - Blending hospital/community } \\
\text { expertise }\end{array}$ & $\begin{array}{l}\text { - For profit company limits access } \\
\text { to charitable funds }\end{array}$ \\
\hline $\begin{array}{l}\text { - Regionalization of specialized } \\
\text { services (ie, malaria) }\end{array}$ & \\
\hline - Cost effective & \\
\hline $\begin{array}{l}\text { - Enhanced academic } \\
\text { teaching/research opportunities }\end{array}$ & \\
\hline $\begin{array}{l}\text { - Regional database major } \\
\text { advantage for utilization, } \\
\text { epidemiology, outcomes research }\end{array}$ & \\
\hline $\begin{array}{l}\text { - High volume, regional service } \\
\text { allows comprehensive technology } \\
\text { assessment to be efficient }\end{array}$ & \\
\hline - Access to capital funds & \\
\hline - For profit business & \\
\hline $\begin{array}{l}\text { - Growth into new markets } \\
\text { expected }\end{array}$ & \\
\hline
\end{tabular}

traregional sites. The laboratory service delivery model has strengthened the region's ability to trend and track antibiotic resistant organisms not only in individual patients, but also in the populace at large, and to have an accurate picture of the incidence of particular types of resistance in different care sectors (ie, hospitals versus the community or long term care facilities). The laboratory has also become a major resource for not only epidemiological but also health outcomes and utilization research projects. Due to the high volume and unique complexity of tests performed by CLS (approximately 2000 to $2200 /$ day), it is also an ideal environment in which to perform technology assessment projects. CLS already has established a track record for obtaining industry funding to support technology assessment projects including running trials for new projects to collect clinical performance data for companies applying for licences to government agencies such as the United States Food and Drug Administration. Finally, CLS has established a research and development office that provides administrative support to externally funded projects that require clinical laboratory work. A microbiology technologist is also employed full time to coordinate externally funded projects (ie, clinical trials, peer-reviewed research, etc) and perform internal research projects.

Although CLS's current space limitations have not allowed the implementation of a full range of continuing medical education activities for staff, other academic teaching commitments have been enhanced. Medical students, residents and fellows regularly rotate through the centralized laboratory 
just as they would have through the laboratories in the academic teaching hospitals. Due to the volume and variety of clinical material handled by CLS microbiology, the laboratory experience of medical trainees, even during shorter rotations, has been expanded. In this environment, previously unusual events have also become more common (ie, isolation of a previously unknown bacterial species). Trainees can also readily complete a comprehensive laboratory project within one to two months because a large amount of clinical material, isolates and data is readily available. Trainees also attend regular formal teaching rounds, which are held at the medical school. The Infectious Diseases Research Group at the University of Calgary comprised most infectious diseases clinicians, medical microbiologists from CLS and other investigators with collaborative interests in infectious diseases research. The Infectious Diseases Research Group has established a formal round schedule that includes lectures by members of the research group or invited speakers and weekly case presentations. One case round a month is devoted to medical microbiology, and one lecture slot is a collaborative journal club where trainees select, present and analyze papers from appropriate journals.

\section{CONCLUSIONS}

In Calgary, microbiology laboratory restructuring is almost complete. Our post-hoc analysis of the major pros and cons of developing and implementing a regional centralized microbiology service are outlined in Table 4. Although it was not clear at the onset, three years after embarking on the implementation plan, the pros substantially outweigh the cons. As consolidation proceeded, many of the cons that were due to a lack of infrastructure have been resolved. The biggest infrastructure problem remains the lack of an adequate laboratory space to support the growth and expansion of the future service needs of the region. However, this situation will be rectified when CLS's new high volume laboratory facility is built in the next two years.

Although the CLS operating budget is capped, revenue can be sought from other sources of funding besides public health care regions or the government because the laboratory is run as a business. Access to capital funds for laboratory equipment in the CLS partnership has been outstanding compared with the previous situation in the public sector. The centralized laboratory offers an exciting opportunity for enhanced academic activity. Centralization of services has allowed us to attract industry support for technology assessment initiatives, not only for new equipment, but also new assays, reagents and supplies. The large, collated database that results from a regionalized microbiology service also creates an environment that is ideal for studying the epidemiology of many infectious diseases problems. For example, the CLS database coordinates accurate information about the incidence of antibiotic-resistant organisms in all care sectors in Calgary. In addition, nosocomial as well as community-based outbreaks are more readily detected.

Although major improvements have occurred in the communication between the centralized laboratory service and its users, face to face interaction with clinicians cannot be easily replaced. New ways of effectively communicating and providing medical consultation must be sought. Rapid advances in computer technology, particularly via the Internet, may soon allow proliferation of telepathology. In the new millenium, CLS microbiology services would like to be able to provide physicians with electronically transmitted reports that contain pictures of the microscopic smear results and even video clips.

Centralization of microbiology services in Calgary was not an easy task. Our success relied on the sustained commitment and efforts of an empowered team of medical, technical and support staff. We have learned a tremendous amount from our successes and mistakes along the way. CLS would be happy to assist other regions that are embarking on a similar restructuring initiative of microbiology services.

\section{REFERENCES}

1. Gibb AP, Karn E. Limited yield from the fifth day of incubation of BacT/Alert standard bottles. American Society for Microbiology, Miami Beach, Florida, May 4-8, 1997. (Abst C-424)

2. Bashshur RL. On the definition and evaluation of telemedicine. Telemed J 1995;1:19-30.

3. Gigsby J, Sanders JH. Telemedicine: Where it is and where it's going. Ann Intern Med 1998;129:123-7.

4. Peredmia DA, Allen A. Telemedicine technology and clinical applications. JAMA 1995;273:483-8.

5. Dunn BE, Ulmagro UA, Choi H, Poecia DL, Weinstein RS. Use of telepathology for routine surgical pathology review in a test bed in the Department of Veteran Affairs. Telemed J 1997;3:1-10. 


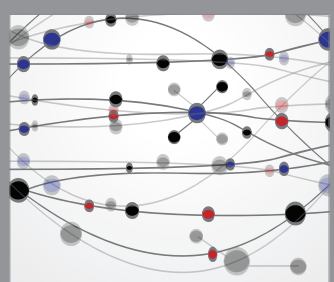

The Scientific World Journal
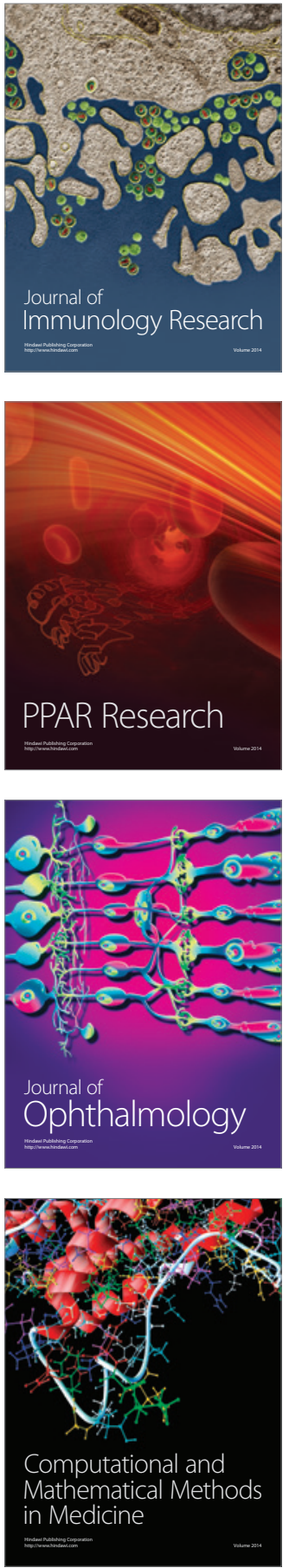

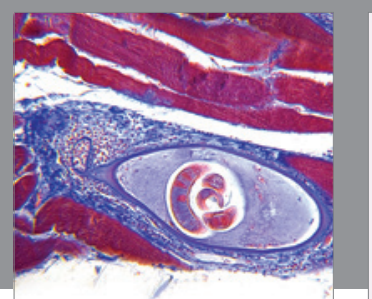

Gastroenterology Research and Practice

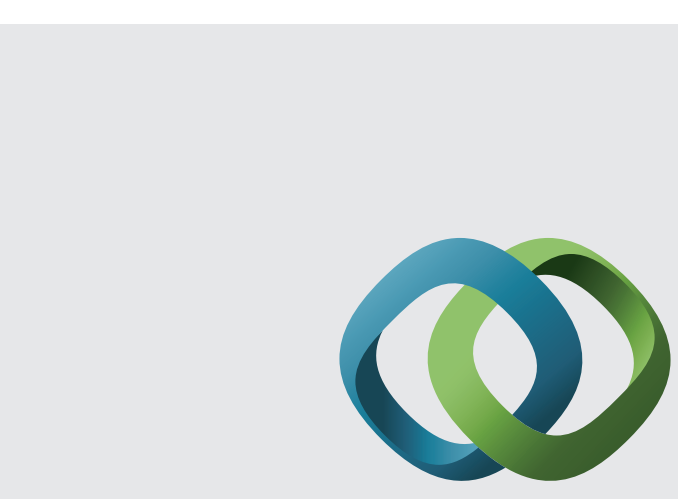

\section{Hindawi}

Submit your manuscripts at

http://www.hindawi.com
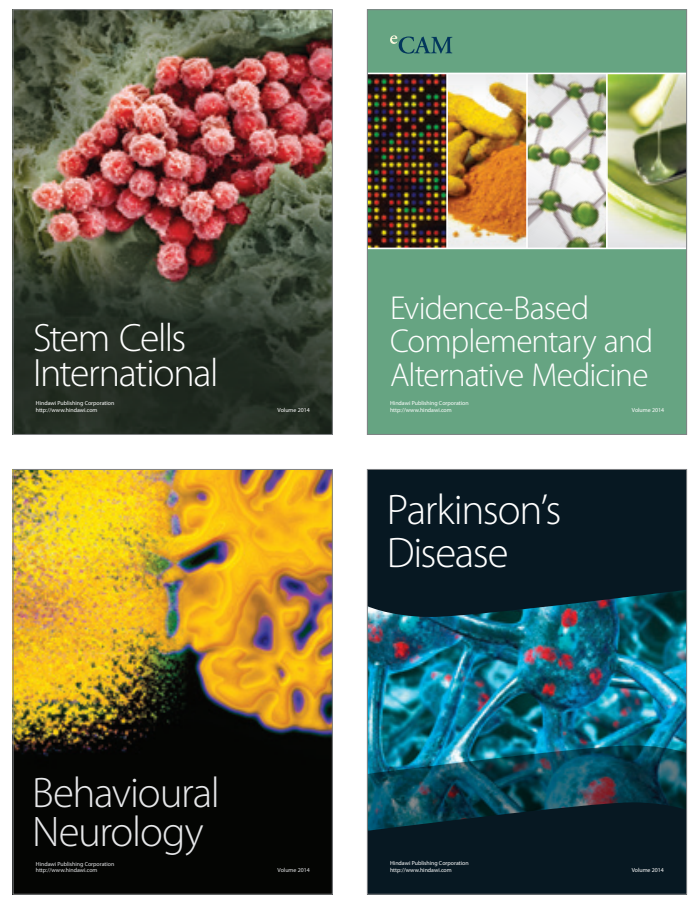
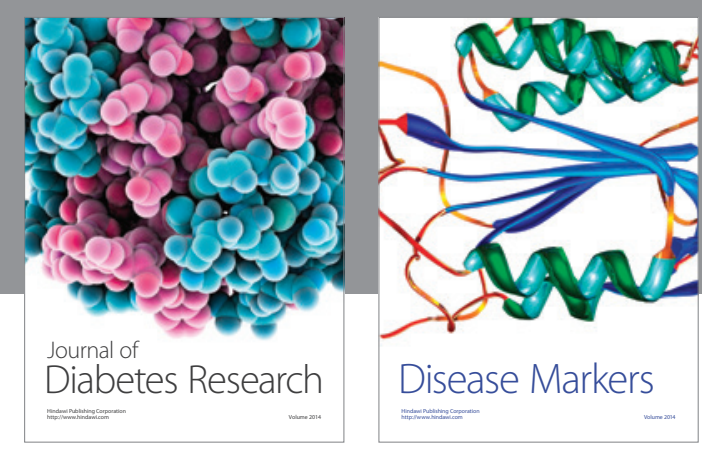

Disease Markers
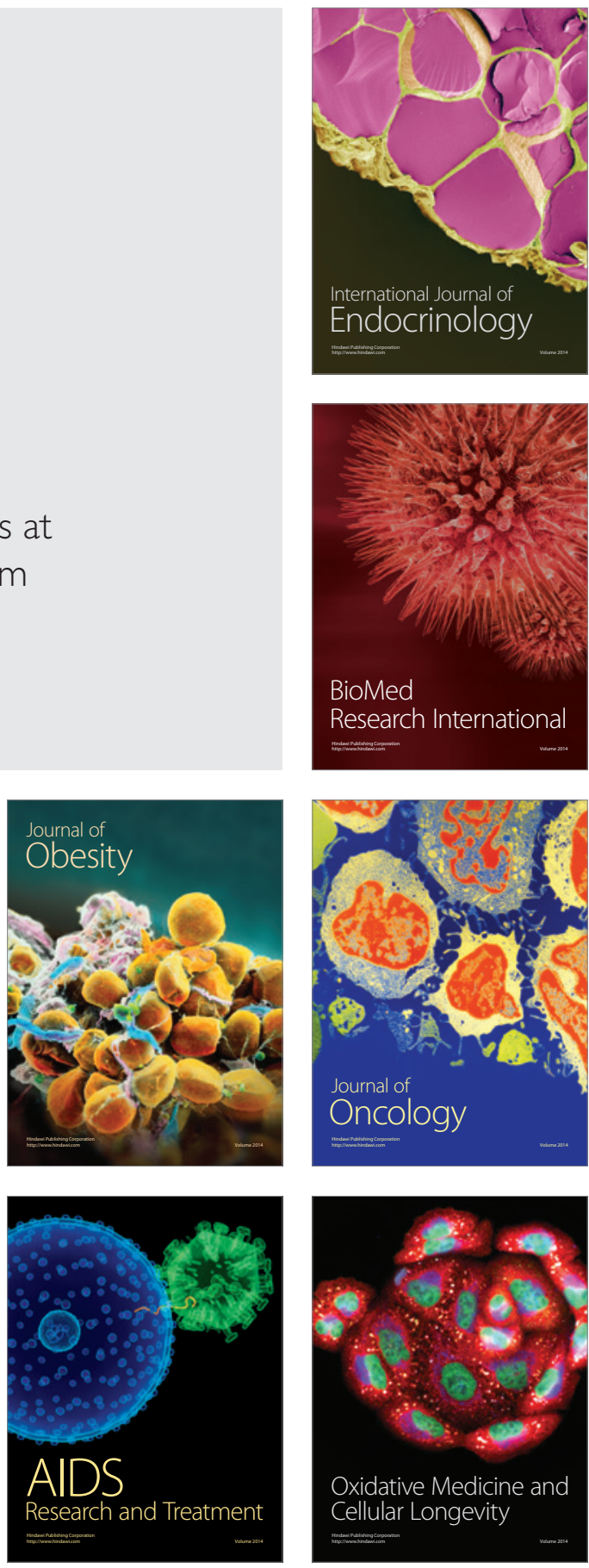